Bull. Korean Math. Soc. 50 (2013), No. 5, pp. 1623-1630

http://dx.doi.org/10.4134/BKMS.2013.50.5.1623

\title{
ON PERIODIC P-CONTINUED FRACTION HAVING PERIOD LENGTH ONE
}

\author{
Amara Chandoul, Hela Ben Amar, and Mohamed Mkaouar \\ Abstract. The aim of this paper is to prove that every quadratic for- \\ mal power series $\omega$ can be expressed as a periodic non-simple continued \\ fraction having period length one.
}

\section{Introduction}

Let $p$ be a prime, $q$ be a power of $p$ and $\mathbb{F}_{q}$ be a field with $q$ elements. Let $\mathbb{F}_{q}\left(\left(X^{-1}\right)\right)$ denote the field of all formal power series $\omega=\sum_{n \geq v} \omega_{n} X^{-n}$ in an indeterminate $X$, with $\omega_{n}$ all lying in the field $\mathbb{F}_{q}$. Recall that $\mathbb{F}_{q}[X]$ denote the ring of polynomials in $X$ with coefficients in $\mathbb{F}_{q}$.

For the above formal Laurent series $\omega$, we may assume that $v \neq 0$. Then the integer $v=v(\omega)$ is called the order of $\omega$. The valuation of $\omega$ is defined to be $|\omega|=q^{-v(\omega)}$. It is well known that $|\cdot|$ is a non-archimedean valuation on the field $\mathbb{F}_{q}\left(\left(X^{-1}\right)\right)$ and $\mathbb{F}_{q}\left(\left(X^{-1}\right)\right)$ is a complete metric space under the metric $\rho$ defined by $\rho(\omega-\psi)=|\omega-\psi|$.

For $\omega=\sum_{n \geq n_{0}} \omega_{n} X^{-n} \in \mathbb{F}_{q}\left(\left(X^{-1}\right)\right)$, let $[\omega]=\sum_{v \leq n \leq 0} \omega_{n} X^{-n} \in \mathbb{F}_{q}[X]$. We call $[\omega]$ the polynomial part of $\omega$. It is evident that the integer $-v(\omega):=-v$ is equal to the degree $\operatorname{deg}[\omega]$ of the polynomial $[\omega]$ provided $v \leq 0$, i.e., $\omega \neq 0$.

Let $D(0,1)$ denote the valuation ideal $X^{-1} \mathbb{F}_{q}\left[\left[X^{-1}\right]\right]$ in the ring of formal power series $\mathbb{F}_{q}\left[\left[X^{-1}\right]\right]$. It consists of all formal series $\sum_{n>1} \omega_{n} X^{-n}$.

Let $P \in \mathbb{F}_{q}[X]$. Consider the following transformation from $D(0,1)$ to $D(0,1)$ defined by

$$
T_{P}(\omega):=\left\{\frac{P}{\omega}\right\}, T_{P}(0):=0 .
$$

This map describes the $P$-continued fraction over the field of Laurent series. As in the classical theory, every $\omega \in D(0,1)$ has the following $P$-continued

Received September 23, 2012.

2010 Mathematics Subject Classification. 11A55, 11D45, 11D72, 11J61, 11J66.

Key words and phrases. continued fraction, Laurent series, finite. 
fraction expansion

$$
\omega=C_{0}+\frac{P}{C_{1}+\frac{P}{C_{2}+\frac{P}{C_{3}+\cdot+\frac{P}{C_{i}+\ddots}}}}=\left[0 ; C_{1}(\omega), C_{2}(\omega), \ldots\right]_{P},
$$

where the digits $C_{i}(\omega)$ are polynomials of strictly positive degree and are defined by

It is clear that

$$
\forall i \geq 1, C_{i}(\omega)=\left[\frac{P}{T_{P}^{i}\left(\frac{P}{\omega}\right)}\right]
$$

$$
T_{P}\left[0, C_{1}, \ldots, C_{i}, \ldots\right]_{P}=\left[0, C_{2}, \ldots, C_{i}, \ldots\right]_{P} .
$$

It is easy to convert a simple continued fraction to a $P$-continued fraction as follows

$$
\left[D_{0}, D_{1}, D_{2}, D_{3}, \ldots\right]=\left[D_{0}, D_{1}, D_{2}, D_{3}, \ldots\right]_{1}=\left[D_{0}, P D_{1}, D_{2}, P D_{3}, \ldots\right]_{P} .
$$

In [1] Burger et al. prove that every real quadratic irrational $\alpha$ can be expressed as a periodic non-simple continued fraction having period length one. Moreover, it is proved that the sequence of rational numbers generated by successive truncations of this expansion is a sequence of convergents of $\alpha$, For further references on the subject, see also [3], [2] and [4].

In this paper, we extend their results over the field of formal power series by proving that every quadratic formal power series $\omega$ can be expressed as a nonsimple continued fraction having period length one. We establish our result by extending some of the arguments of [1], in this new context and making appropriate adjustments.

\section{Results}

Each $\omega \in \mathbb{F}_{q}\left(\left(X^{-1}\right)\right)$ has a continued fraction representation given by $\omega=$ $\left[a_{0} ; a_{1}, \ldots\right], \operatorname{deg} a_{i} \geq 1, \forall i \geq 1$, we refer to the $\left(\frac{A_{n}}{B_{n}}\right)_{n \geq 0}$ as convergents to $\omega$.

$A_{n}$ and $B_{n}$ satisfy the recurrence relation:

$$
\begin{gathered}
A_{-1}=1, A_{0}=D_{0}, A_{n}=D_{n} A_{n-1}+A_{n-2} \text { for } n=1,2, \ldots, \\
B_{-1}=0, B_{0}=1, \quad B_{n}=D_{n} B_{n-1}+B_{n-2} \text { for } n=1,2, \ldots, \\
A_{n} B_{n-1}-B_{n} A_{n-1}=(-1)^{n} \text { for } n=1,2, \ldots
\end{gathered}
$$

Now, we give the main result of this section. 
ON PERIODIC P-CONTINUED FRACTION HAVING PERIOD LENGTH ONE 1625

Theorem 2.1. Let $\omega$ an arbitrary quadratic formal power series written as

$$
\omega=\left[a_{0}, \ldots, a_{s-1}, \overline{D_{0}, D_{1}, \ldots, D_{t-1}}\right] .
$$

Then $\omega$ can be expressed as the period one $(-1)^{t} R_{t+1}^{2}$-continued fraction

$$
\begin{gathered}
\omega=\left[a_{0},(-1)^{t} R_{t+1}^{2} a_{1}, \ldots,(-1)^{t} R_{t+1}^{2} a_{s-1},\right. \\
\left.D_{0},(-1)^{t+1} R_{t+1} B_{t+1}, \overline{(-1)^{t+1} R_{t+1} P}\right]_{(-1)^{t} R_{t+1}^{2}},
\end{gathered}
$$

where $P=A_{t}+B_{t-1}$ and $R_{t+1}=D_{0} B_{t+1}-A_{t+1}$.

Remark 2.2. If the preperiod of $\omega$ is odd, then $\omega=\left[a_{0}, \ldots, a_{s-1}, \overline{D_{0}, D_{1}, \ldots, D_{t-1}}\right]=\left[a_{0}, \ldots, a_{s-1}, D_{0}, \overline{D_{1}, \ldots, D_{t-1}, D_{0}}\right]$.

Consequently, we can suppose that $s$ is even in Theorem 2.1.

In order to prove this theorem, we need the following lemmas.

Lemma 2.3. Let $R_{i}=D_{0} B_{i}-A_{i}$ for all $i \geq 0$. For all $t$, the following assertions are satisfied:

(i) $R_{t-1} A_{t}+R_{t-2} B_{t}=\left(B_{t-2}+A_{t-1}\right) R_{t}$.

(ii) $B_{t-1} R_{t-2}-R_{t-1} B_{t-2}=(-1)^{t}$.

(iii) $A_{t-1} R_{t-2}-R_{t-1} A_{t-2}=D_{0}(-1)^{t}$.

(iv) $D_{0} R_{t-1}+R_{t-2}=R_{t}$.

Proof. Replacing $R_{i}$ by $D_{0} B_{i}-A_{i}$ and using (2.3), we get.

(i) $\quad R_{t-1} A_{t}+R_{t-2} B_{t}-\left(B_{t-2}+A_{t-1}\right) R_{t}$

$$
\begin{aligned}
& =D_{0}\left(A_{t} B_{t-1}-A_{t-1} B_{t}\right)+\left(A_{t} B_{t-2}-A_{t-2} B_{t}\right) \\
& =D_{0}(-1)^{t-1}+D_{0}(-1)^{t-2} \\
& =0 .
\end{aligned}
$$

(ii) $B_{t-1} R_{t-2}-R_{t-1} B_{t-2}=-B_{t-1} A_{t-2}+A_{t-1} B_{t-2}=(-1)^{t}$.

(iii) $A_{t-1} R_{t-2}-R_{t-1} A_{t-2}=D_{0}\left(A_{t-1} B_{t-2}-B_{t-1} A_{t-2}\right)=D_{0}(-1)^{t}$.

(iv) $D_{0} R_{t-1}+R_{t-2}=D_{0}\left(D_{0} B_{t-1}+B_{t-2}\right)-\left(D_{0} A_{t-1}+A_{t-2}\right)$,

$$
\begin{aligned}
& =D_{0} B_{t}-A_{t} \\
& =R_{t} .
\end{aligned}
$$

Lemma 2.4. Let $\omega=\left[\overline{D_{0}, D_{1}, \ldots, D_{t-1}}\right]$ be a quadratic formal power series having a purely simple continued fraction of period of length $t$. Let $\left(\frac{A_{n}}{B_{n}}\right)_{n}$ be the sequence of the convergents of $\omega, P=A_{t}+B_{t-1}$ and

$$
R_{i}=D_{0} B_{i}-A_{i} \text { for all } i \geq 0 \text {. }
$$

Then

$$
\omega=\frac{D_{0} \varphi_{P}-R_{t-1}}{\varphi_{P}+R_{t}}
$$

where $\varphi_{P}=\varphi(P, t)$ is the root of $Y^{2}-P Y+(-1)^{t+1}=0$, with $\left[\varphi_{P}\right]=P$. 
Proof. Let $\omega=\left[\overline{D_{0}, D_{1}, \ldots, D_{t-1}}\right]$. Then $\omega$ is the unique root of the equation

$$
\Lambda(Y)=B_{t} Y^{2}+\left(B_{t-1}-A_{t}\right) Y-A_{t-1}
$$

such that $|\omega|>1$. Let $\gamma=\frac{D_{0} \varphi_{P}-R_{t-1}}{\varphi_{P}+R_{t}}$, since $|\gamma|>1$, then it is sufficient to show that $\Lambda(\gamma)=0$ in order to prove that $\gamma=\omega$. We have

$$
\begin{aligned}
\Lambda(\gamma) & =B_{t}\left(\frac{D_{0} \varphi_{P}-R_{t-1}}{\varphi_{P}+R_{t}}\right)^{2}+\left(B_{t-1}-A_{t}\right)\left(\frac{D_{0} \varphi_{P}-R_{t-1}}{\varphi_{P}+R_{t}}\right)-A_{t-1} \\
& =\frac{\alpha \varphi_{P}^{2}+\beta \varphi_{P}+\lambda}{\left(\varphi_{P}+R_{t}\right)^{2}}
\end{aligned}
$$

where, according to $(2.2)$,

$$
\begin{aligned}
\alpha & =B_{t} D_{0}^{2}+D_{0}\left(B_{t-1}-A_{t}\right)-A_{t-1} \\
& =D_{0}\left(D_{0} B_{t}+B_{t-1}\right)-\left(D_{0} A_{t}+A_{t-1}\right) \\
& =D_{0} B_{t+1}-A_{t+1} \\
& =R_{t+1} \\
\beta & =-2 D_{0} R_{t-1} B_{t}+\left(B_{t-1}-A_{t}\right)\left(D_{0} R_{t}-R_{t-1}\right)-2 A_{t-1} R_{t} \\
& =R_{t-1}\left(-2 D_{0} B_{t}-B_{t-1}+A_{t}\right)+R_{t}\left(D_{0}\left(B_{t-1}-A_{t}\right)-2 A_{t-1}\right) \\
& =R_{t-1}\left(-B_{t+1}-R_{t}\right)+R_{t}\left(R_{t-1}-A_{t+1}\right) \\
& =-R_{t-1} B_{t+1}-R_{t} A_{t+1} .
\end{aligned}
$$

Using Lemma 2.3(i), we obtain

$$
\beta=-\left(B_{t-1}+A_{t}\right) R_{t+1}=-P R_{t+1},
$$

and

$$
\begin{aligned}
\lambda & =B_{t} R_{t-1}^{2}-\left(B_{t-1}-A_{t}\right) R_{t} R_{t-1}-A_{t-1} R_{t}^{2}, \\
& =R_{t-1}\left(B_{t} R_{t-1}-R_{t} B_{t-1}\right)+R_{t}\left(A_{t} R_{t-1}-A_{t-1} R_{t}\right) .
\end{aligned}
$$

Lemma 2.3(ii), (iii) and (iv) implies that

$$
\begin{aligned}
\lambda & =R_{t-1}(-1)^{t+1}+R_{t}(-1)^{t+1} D_{0}, \\
& =(-1)^{t+1}\left(D_{0} R_{t}+R_{t-1}\right), \\
& =(-1)^{t+1} R_{t+1} .
\end{aligned}
$$

Finally,

$$
\Lambda(\gamma)=\frac{R_{t+1}\left(\varphi_{P}^{2}-P \varphi_{P}+(-1)^{t+1}\right)}{\left(\varphi_{P}+R_{t}\right)^{2}}=0 .
$$

Lemma 2.5. Let $\omega \in \mathbb{F}_{q}\left(\left(X^{-1}\right)\right)$ such that $\omega=\left[\overline{D_{0}, D_{1}, \ldots, D_{t-1}}\right]$ and $\left(\frac{A_{n}}{B_{n}}\right)_{n}$ be the sequence of the convergents of $\omega$. Then

$$
\omega=\left[D_{0}, B_{t+1}, \overline{(-1)^{t+1} R_{t+1} P, P}\right]_{-R_{t+1}}
$$

and

$$
\omega=\left[D_{0},(-1)^{t+1} R_{t+1} B_{t+1}, \overline{(-1)^{t+1} R_{t+1} P}\right]_{(-1)^{t} R_{t+1}^{2}},
$$



where $P=A_{t}+B_{t-1}$.

Proof. Let $\omega=\left[\overline{D_{0}, D_{1}, \ldots, D_{t-1}}\right]$. Then it is clear, by Lemma 2.4 that

$$
\omega=\frac{D_{0} \varphi_{P}-R_{t-1}}{\varphi_{P}+R_{t}}
$$

where $\varphi_{P}$ is the root of $Y^{2}-P Y+(-1)^{t+1}=0$, with $P=A_{t}+B_{t-1}$ and $\left[\varphi_{P}\right]=P$. Then, we have

$$
\omega=D_{0}+\frac{-R_{t+1}}{B_{t+1}+\frac{(-1)^{t}}{\varphi_{P}}} .
$$

As $\varphi_{P}=P+\frac{(-1)^{t}}{\varphi_{P}}$, we get

$$
\begin{aligned}
& \omega=D_{0}+\frac{-R_{t+1}}{B_{t+1}+\frac{(-1)^{t}}{\varphi_{P}}} \\
& =D_{0}+\frac{-R_{t+1}}{B_{t+1}+\frac{-R_{t+1}}{(-1)^{t+1} R_{t+1} P+\frac{-R_{t+1}}{P+\frac{(-1)^{t}}{\varphi_{P}}}}} \\
& =D_{0}+\frac{-R_{t+1}}{B_{t+1}+\frac{-R_{t+1}}{(-1)^{t+1} R_{t+1} P+\frac{-R_{t+1}}{P+\frac{(-1)^{t}}{\varphi_{P}}}}} \\
& =D_{0}+\frac{-R_{t+1}}{B_{t+1}+\frac{-R_{t+1}}{(-1)^{t+1} R_{t+1} P+\frac{-R_{t+1}}{P+\frac{-R_{t+1}}{(-1)^{t+1} R_{t+1} P+\frac{-R_{t+1}}{\varphi_{P}}}}}} \\
& =\left[D_{0}, B_{t+1}, \overline{(-1)^{t+1} R_{t+1} P, P}\right]_{-R_{t+1}} .
\end{aligned}
$$

In addition, we have

$$
\omega=D_{0}+\frac{-R_{t+1}}{B_{t+1}+\frac{-R_{t+1}}{(-1)^{t+1} R_{t+1} P+\frac{-R_{t+1}}{P+\frac{-R_{t+1}}{(-1)^{t+1} R_{t+1} P+\frac{-R_{t+1}}{\varphi_{P}}}}}}
$$




$$
\begin{aligned}
& =D_{0}+\frac{(-1)^{t} R_{t+1}^{2}}{(-1)^{t+1} R_{t+1} B_{t+1}+\frac{(-1)^{t} R_{t+1}^{2}}{(-1)^{t+1} R_{t+1} P+\frac{(-1)^{t} R_{t+1}^{2}}{(-1)^{t+1} R_{t+1} P+\frac{(-1)^{t} R_{t+1}^{2}}{\varphi_{P}}}}} \\
& =\left[D_{0},(-1)^{t+1} R_{t+1} B_{t+1}, \overline{(-1)^{t+1} R_{t+1} P}\right]_{(-1)^{t} R_{t+1}^{2}} \cdot
\end{aligned}
$$

Proof of Theorem 2.1. Let

$$
\omega=\left[a_{0}, \ldots, a_{s-1}, \overline{D_{0}, D_{1}, \ldots, D_{t-1}}\right] \text { and } \omega^{*}=\left[\overline{D_{0}, D_{1}, \ldots, D_{t-1}}\right] .
$$

By Lemma 2.5 and (2.6), we conclude that

$$
\omega^{*}=\left[D_{0},(-1)^{t+1} R_{t+1} B_{t+1}, \overline{(-1)^{t+1} R_{t+1} P}\right]_{(-1)^{t} R_{t+1}^{2}} .
$$

Applying (1.3) to $\omega$, we obtain

$$
\omega=\left[a_{0},(-1)^{t} R_{t+1}^{2} a_{1}, \ldots,(-1)^{t} R_{t+1}^{2} a_{s-1}, \omega^{*}\right] .
$$

We have immediately from (2.7) and (2.8),

$$
\begin{aligned}
\omega=[ & a_{0},(-1)^{t} R_{t+1}^{2} a_{1}, \ldots,(-1)^{t} R_{t+1}^{2} a_{s-1}, \\
& \left.D_{0},(-1)^{t+1} R_{t+1} B_{t+1}, \overline{(-1)^{t+1} R_{t+1} P}\right]_{(-1)^{t} R_{t+1}^{2}} .
\end{aligned}
$$

\section{Examples}

Example 3.1. Let $\omega \in \mathbb{F}_{2}\left(\left(X^{-1}\right)\right)$ be a quadratic formal power series solution of the equation

$$
\left(X^{2}+X+1\right) Y^{2}+\left(X^{3}+X^{2}+X+1\right) Y+X^{2}+X+1=0 .
$$

We can prove that the continued fraction expansion of $\omega$ is defined as follows

$$
\omega=[\overline{X, X+1, X}] .
$$

Applying Lemma 2.5, we obtain

$$
\omega=\left[X, R_{4} B_{4}, \overline{R_{4} P}\right]_{-R_{4}^{2}},
$$

where $P=A_{3}+B_{2}$.

Using the induction formulas (2.1) and (2.2), we obtain

$$
\begin{aligned}
& A_{1}=X^{2}+X+1 \\
& A_{2}=X^{2}(X+1) \\
& A_{3}=X^{3}(X+1)+X(X+1)+1 \\
& A_{4}=\left(X^{3}+1\right)\left(X^{2}+1\right)
\end{aligned}
$$


and

$$
\begin{aligned}
& B_{1}=X+1 \\
& B_{2}=X^{2}+X+1 \\
& B_{3}=X^{3}+X^{2}+1 \\
& B_{4}=X^{4}
\end{aligned}
$$

Then

$$
\begin{aligned}
R_{4} & =D_{0} B_{4}-A_{4} \\
& =X^{3}+X^{2}+1
\end{aligned}
$$

and

$$
P=X^{3}(X+1)
$$

Finally

$$
\omega=\left[X, X^{7}+X^{6}+X^{4}, \overline{X^{7}+X^{6}+X^{3}+X}\right]_{X^{6}+X^{4}+1} .
$$

Example 3.2. Let $\psi \in \mathbb{F}_{2}\left(\left(X^{-1}\right)\right)$ be a quadratic formal power series solution of the equation

$$
\left(X^{2}+X+1\right) Y^{2}+X^{3} Y+X^{3}+X^{2}+1=0 .
$$

We can prove that the continued fraction expansion of $\psi$ is defined as follows

$$
\psi=\left[X, X^{2}+1, X^{2}, \overline{X+1, X, X+1}\right] .
$$

The preperiod of $\psi$ is odd, so by Remark 2.2

$$
\psi=\left[X, X^{2}+1, X^{2}, X+1, \overline{X, X+1, X}\right] .
$$

Let $\psi^{*}=[\overline{X, X+1, X}]$. Then $\psi=\left[X, X^{2}+1, X^{2}, X+1, \psi^{*}\right]$, and by Example 3.1

$$
\psi^{*}=\left[X, X^{7}+X^{6}+X^{4}, \overline{X^{7}+X^{6}+X^{3}+X}\right]_{X^{6}+X^{4}+1} .
$$

Applying Theorem 2.1, we obtain

$\psi=\left[X,\left(X^{2}+1\right) L, X^{2} L,(X+1) L, X, X^{7}+X^{6}+X^{4}, \overline{X^{7}+X^{6}+X^{3}+X}\right]_{L}$,

where $L=X^{6}+X^{4}+1$.

\section{References}

[1] E. B. Burger, J. Gell-Redman, R. Kravitz, D. Walton, and N. Yates, Shrinking the period lengths of continued fractions while still capturing convergents, J. Number Theory $\mathbf{1 2 8}$ (2008), no. 1, 144-153.

[2] E. B. Van Vleck, On the convergence of the continued fraction of Gauss and other continued fractions, Ann. of Math. (2) 3 (1901/02), no. 1-4, 1-18.

[3] - On the convergence of algebraic continued fractions whose coefficients have limiting values, Trans. Amer. Math. Soc. 5 (1904), no. 3, 253-262.

[4] H. Waadeland, Tales about tails, Proc. Amer. Math. Soc. 90 (1984), no. 1, 57-64. 
Amara Chandoul

FACUlté des Sciences de Sfax

BP 1171, SFAx 3000, Tunisia

E-mail address: amarachandoul@yahoo.fr

Hela Ben Amar

Faculté des Sciences de Sfax

BP 1171, SFAx 3000, Tunisia

E-mail address: Hela.BenAmar@issatgb.rnu.tn

Mohamed Mkaouar

Faculté des Sciences de Sfax

BP 1171, SFAx 3000, Tunisia

E-mail address: mohamed.mkaouar@fss.rnu.tn 